\title{
Comparison of automated procedures for ARMA model identification
}

\author{
Tetiana Stadnytska, Simone Braun, and Joachim Werner \\ University of Heidelberg, Heidelberg, Germany
}

\begin{abstract}
This article evaluates the performance of three automated procedures for ARMA model identification commonly available in current versions of SAS for Windows: MINIC, SCAN, and ESACF. Monte Carlo experiments with different model structures, parameter values, and sample sizes were used to compare the methods. On average, the procedures either correctly identified the simulated structures or selected parsimonious nearly equivalent mathematical representations in at least $60 \%$ of the trials conducted. For autoregressive models, MINIC achieved the best results. SCAN was superior to the other two procedures for mixed structures. For moving-average processes, ESACF obtained the most correct selections. For all three methods, model identification was less accurate for low dependency than for medium or high dependency processes. The effect of sample size was more pronounced for MINIC than for SCAN and ESACF. SCAN and ESACF tended to select higherorder mixed structures in larger samples. These findings are confined to stationary nonseasonal time series.
\end{abstract}

Time series analysis deals with repeated and equally spaced observations on a single unit. Classical statistical techniques are no longer appropriate here because data points cannot be assumed independent and uncorrelated. One of the most widely employed procedures for time series data is the autonegressive integrated moving-average (ARIMA) approach proposed by Box and Jenkins (1970). Since Glass, Willson, and Gottman (1975) introduced the ARIMA technique to the social and behavioral sciences, this methodology has been increasingly employed in different research fields (Delcor, Cadopi, Delignières, \& Mesure, 2002; Fortes, Ninot, \& Delignières, 2005; McCleary \& Hay, 1980; Ninot, Fortes, \& Delignières, 2005; Velicer \& Colby, 1997; Velicer \& Fava, 2003; Wagenmakers, Farrell, \& Ratcliff, 2004).

The idea behind the Box-Jenkins procedure is to infer the true data generating process from an observed time series. The ARIMA strategy is based on a three-step iterative cycle of model identification, model estimation, and diagnostic checks in model accuracy. (For detailed treatments of the technique, see Bowerman \& O'Connell, 1993; Box, Jenkins, \& Reinsel, 1994; Brockwell \& Davis, 2002; and Makridakis, Wheelwright, \& Hyndman, 1998.) Model identification step provides insight into properties of the underlying stochastic process of the variable under study. Once the process has been inferred, it can be used either to test some hypothesis about its generating mechanism, forecast future values of the series, or remove dependency from the data so that it meets assumptions of the general linear model, as in interrupted time series experiments. In the first two cases, accurate model selection is indispensable.

Especially in theory testing, where model identification represents the primary goal of the analysis, model mis- specification implies serious conceptual consequences and should be omitted. For instance, autoregressive models are characteristic of behavior containing internal temporal regularity, whereas moving-average models are characteristic of systems depending on external and occasional events. Thus autoregressive patterns are typically found in the studies investigating addictive behaviors (Rosel \& Elósegui, 1994; Velicer, Redding, Richmond, Greeley, \& Swift, 1992; Velicer, Rossi, Diclemente, \& Prochaska, 1996). For nonaddictive habits of occasional smokers or drinkers, however, moving-average models are more appropriate. Analyzing travel behavior of different population groups, Fraschini and Axausten (2001) found that autoregressive models predominated in the age classes between 35 and 65 years old, reflecting more regular behavior probably caused by fixed employment and a more settled lifestyle. In contrast, moving-average patterns were mainly identified for younger participants, indicating the predominance of external influences on subject behavior. According to Velicer and Fava (2003), three popular hypotheses concerning nicotine's role in maintaining smoking, such as the fixed effect, nicotine regulation, and multiple regulation models, imply competing ARIMA patterns.

When the goal of research is to determine the efficacy of a specific intervention, as in interrupted time series analysis, model identification represents an intermediate step serving to transform the data (Glass et al., 1975). Hence, accurate model selection is not crucial here. Several alternative procedures avoiding model identification have been proposed in the last three decades (Algina \& Swaminathan, 1977, 1979; Crosbie, 1993; Gottman, 1981; McKnight, McKean, \& Huitema, 2000; Simonton, 1977; Velicer \& McDonald, 1984, 
1991). The approach of Algina and Swaminathan (1977, 1979) requires that the number of units exceed the number of observations across time, thus it is inappropriate in most applied research settings. Simonton's (1977) method and the general transformation approach of Velicer and McDonald $(1984,1991)$ assuming autoregressive model with one to five parameters for the data performed well in evaluation studies (Harrop \& Velicer, 1985, 1990) and can be implemented with most existing statistic software packages. Two popular methods for interrupted time series analysis, ITSE (Gottman, 1981; Williams \& Gottman, 1999) and ITSACORR (Crosbie, 1993), have been recently strongly criticized by Huitema $(2002,2004)$, who demonstrated that both techniques yield greatly distorted intervention effect parameter estimates. Huitema recommends avoiding these procedures and offers the double bootstrap method of McKnight et al. (2000) as an alternative for sample sizes of $T \leq 50$. (Software for this method is available at www.stat.wmich.edu/slab/Software/ Timeseries.html.) To summarize, in time series experiments, the ARIMA model identification is just one possibility to prewhiten the series, which can be successfully replaced by alternative techniques such as the general transformation approach of Velicer and McDonald $(1984,1991)$ or the double bootstrap method of McKnight et al. (2000).

There are two major approaches for model identification: the traditional Box-Jenkins procedure and time series diagnostics by automated methods (Choi, 1992). The former consists in detailed inspection of empirical autocorrelation and partial autocorrelation functions (ACF and PACF) and in comparing their shape and value with theoretical ARIMA patterns. Model selection using the Box-Jenkins methodology is a sophisticated procedure requiring many data points and a great deal of researcher expertise. Moreover, Velicer and Harrop (1983) demonstrated that even highly trained judges experienced considerable difficulty in identifying models present in computer-generated series: only $28 \%$ of the cases were classified correctly. Therefore, more reliable and less subjective automated methods are the focus of this article.

The automated procedures evaluated in the present study, MINIC, SCAN, and ESACF, were chosen primarily on pragmatic grounds. The methods are available in popular computer packages such as SAS for Windows, so they have become increasingly visible in applied research during the past years. Moreover, in comparison with other automated procedures, the selected methods were less criticized in the time series literature. The performance of MINIC, SCAN, and ESACF was studied on stationary time series because this type of processes is the most common in the behavioral sciences. Before presenting our study, we provide a brief introduction to ARIMA modeling, describe the procedures evaluated, and review empirical findings on the performance of automated methods.

\section{ARIMA Modeling}

The general ARIMA $(p, d, q)$ model is given by the equation

$$
(1-B)^{d} Y_{t}=\mu+\frac{\theta(B)}{\phi(B)} U_{t}, U_{t} \sim \operatorname{IIDN}\left(0, \sigma^{2}\right)
$$

where $\mu$ is the true location of the series at time $t=0, B$ is the backward shift operator, which converts the observation $Y_{t}$ into previous observation $B^{n} Y_{t}=Y_{t-n},(1-B)^{d}$ is a nonstationarity factor, $\phi(B)=1-\phi_{1} B-\phi_{2} B^{2}-\ldots-\phi_{p} B^{p}$ is the autoregressive polynomial, and $\theta(B)=1-\theta_{1} B-$ $\theta_{2} B^{2}-\ldots-\theta_{q} B^{q}$ is the moving-average polynomial.

Therefore, each ARIMA model is characterized by the three parameters: $p, d$, and $q$. The value of the autoregressive parameter $p$ reflects how many preceding observations influence the current observation $Y_{t}$. The value of the moving-average term $q$ describes how many previous random shocks $\left(U_{t}\right)$ must be taken into account to describe the dependency present in the time series. The magnitude of the dependence is quantified by $\phi$ or $\theta$. The parameter $d$ refers to the order of differencing that is necessary to stabilize the time series. In stationary processes, all moments including mean and variance are constant over time. Thus stationary time series are stable and don't require differencing or other transformations. Since there is no integration or $d=0$, stationary ARIMA models can also be termed ARMA. The $(0,0,0)$ process with no dependency is called white noise.

As stated previously, the Box-Jenkins model identification technique consists in detailed inspection of empirical autocorrelation and partial autocorrelation functions and in comparing their shape and value with theoretical ARIMA patterns. This traditional visual analysis is often inaccurate because estimates of ACF and PACF received from finite samples can be rather ambiguous, even under ideal conditions. Furthermore, quality of empirical ACF and PACF crucially depends on the number of observations in a series and is sensitive to outliers or other departures from ideal assumptions. The Box-Jenkins method is not very useful in identifying mixed ARMA models where $p$ and $q$ are unequal 0 . The reason for the difficulty is that the ACF and PACF of mixed models tail off to infinity rather than cut off at a particular lag.

\section{Automated Methods}

Numerous alternatives to the Box-Jenkins approach have been developed during the last three decades to make the model identification process more reliable and less subjective. Choi (1992) published a survey of different procedures for model identification, where automated methods are classified into three categories: penalty function methods (e.g., BIC of Rissannen, 1978, and Schwarz, 1978; AIC of Akaike, 1974), innovation regression methods (e.g., HR of Hannan \& Rissanen, 1982; KP of Koreisha \& Pukkila, 1990), and pattern identification methods (e.g., the corner method of Beguin, Gourieroux, \& Montfort, 1980; ESACF and SCAN of Tsay \& Tiao, 1984, 1985). HR, SCAN, and ESACF are commonly available in current versions of SAS for Windows.

Hannan and Rissanen's method called in SAS minimum information criterion (MINIC) combines the regression technique and the penalty functions AIC and BIC for the modeling of stationary and invertible $\operatorname{ARMA}(p, q)$ processes. The MINIC procedure consists of three steps. The first is to fit a high-order AR model to the observations. The choice of autoregressive parameter is determined by 
the order minimizing the AIC. The second step is to apply the ordinary least squares (OLS) method to the series and estimated innovations of the fitted AR model. As a result, $m$ autoregressive and $j$ moving-average OLS estimates are obtained $\left(m=p_{\min }, \ldots, p_{\max }\right.$ is the autoregressive test order, $j=q_{\min }, \ldots, q_{\max }$ is the moving-average test order). As the last step, the BIC is computed for each of $m \times j$ ARMA models. A model with the smallest BIC is used as a MINIC recommendation. For a detailed treatment of the Hannan and Rissanen method, see Choi (1992), Hannan and Rissanen (1982), and SAS (1999).

The extended sample autocorrelation function (ESACF) and the smallest canonical correlation method (SCAN) proposed by Tsay and Tiao $(1984,1985)$ belong to the pattern identification approach. These procedures can be applied for identifying both stationary and nonstationary models. As the major advantage of using ESACF and SCAN, Tsay and Tiao claimed ability of these methods to recognize mixed ARMA models.

The idea behind the standard ESACF approach is to identify the orders of an ARMA process employing iterated least squares estimates of the autoregressive parameters. First, estimates for the autoregressive components are obtained and removed from the data. Then the order of the remaining moving-average component is determined from the transformed series. Since the order of the autoregressive component is never known in advance, an array of autocorrelations from series for which AR $(m)$ components have been removed must be calculated. The autocorrelations of the filtered series are termed extended sample autocorrelations (ESAC). The order of the time series is tentatively determined from the shape of the zero and nonzero elements in an overall ESAC array. The vertex of the triangle of zero values identifies the order of the process. If an empirical ESACF table contains more than one triangular region in which all elements are insignificant (zero values), SAS gives several recommendations ordered by the number of insignificant terms contained in the triangle. The first recommendation is a model with a maximum triangular pattern. For a detailed description of the ESACF method, see Choi (1992), Tsay and Tiao (1984, 1990), and SAS Institute (1999).

The SCAN method of Tsay and Tiao (1985) employs canonical correlation for ARMA model identification. The procedure consists in analyzing eigenvalues of the correlation matrix of the ARMA process. The first step is to compute the $(m+1) \times(m+1)$ matrix containing covariances and variances of the vectors $\mathbf{y}_{m, t}$ and $\mathbf{y}_{m, t-j-1}$, where $t$ ranges from $j+m+2$ to $T . m=p_{\min }, \ldots, p_{\max }$ is the autoregressive test order, $j=q_{\min }, \ldots, q_{\max }$ is the moving-average test order; and $\mathbf{y}_{m, t}=\left(\widetilde{Y}_{t}, \widetilde{Y}_{t-1}, \ldots, \widetilde{Y}_{t-m}\right)^{\prime}$, where $\widetilde{Y}_{t}$ is a mean corrected series $\widetilde{Y}_{t}=Y_{t}-\mu$ with $1 \leq$ $t \leq T$. The smallest eigenvalue of this matrix serves as the squared canonical correlation estimate for models $(m, \hat{j})$. Finding a rectangular pattern in which canonical correlation estimates are insignificant for all specified test orders ( $m \geq p+d, j \geq q$ ) then identifies the ARMA model. If there is more than one zero rectangular in an empirical SCAN table, parsimony and the number of insignificant items in the rectangular pattern determine the model order. For more details about the SCAN method, consult Choi (1992), Tsay and Tiao (1985), and SAS (1999).

\section{Review of Empirical Findings}

Dickey (2004) compared the performance of MINIC, SCAN, and ESACF on 600 simulated ARMA $(1,1)$ series, each of whose length was $T=500$. SCAN showed the best performance, with 461 correctly identified series. ESACF did slightly worse (441 correct identifications). Both SCAN and ESACF were superior to MINIC (252 correct classifications). The methods almost never underestimated $p$ or $q$ when $T=500$. The same experiment with series length of 50 resulted in 203, 65, and 53 correct identifications for SCAN, ESACF, and MINIC, respectively. Also, the simulation study showed that complexity of the process affected the performance of the identification methods. For 600 replicates of the model with $\phi_{4}=0.5$ and $\theta_{1}=$ -0.3 using $T=50$, the correct model was rarely chosen by any technique. The numbers of correct selections were 10 for MINIC, 52 for ESACF and none for SCAN.

Koreisha and Yoshimoto (1991) conducted Monte Carlo experiments with three identification procedures including ESACF, the corner method, and the autoregressive order determination criterion (ARCRI), a method similar to the HR approach. The ARCRI procedure outperformed the other two methods regardless of model structure. The ESACF method performed poorly regardless of sample size. ESACF showed the tendency to overparameterization. This means the incorrect selection of higher order ARMA structures. The results were better for MA than for AR models. ESACF's power in selecting the order of mixed processes was surprisingly low; the percentage of correct identifications was between 21 and 62. Increasing the number of observations did not improve the performance of the ESACF approach. In many cases, the percentage of correctly identified structures was even higher for smaller sample sizes. Koreisha and Yoshimoto tried to improve the performance of ESACF increasing the confidence interval to \pm 3 standard errors. Using wider confidence intervals ensured better results; the ability of ESACF to identify the real ARMA structure was, however, still below that of the ARCRI procedure.

Chan (1999) compared the performance of ESACF, SCAN, and the Corner method for a mixed $(2,1)$ ARMA model. ESACF yielded the best results: The percentage of correct identifications was 13.0 for $T=100,25.1$ for $T=$ $200,30.2$ for $T=400$, and 33.5 for $T=1,000$ in outlierfree time series. The most frequent incorrect selections were $(1,2)$ and $(1,3)$ models. The performance of SCAN was distinctly worse, with $1.0 \%, 9.5 \%, 18.4 \%$, and $20.3 \%$ of correct selections for $T=100,200,400$, and 1,000, respectively. The most common erroneous identifications of SCAN were $(3,0)$ and $(1,2)$ structures. ESACF was also distinctly superior to the other two methods in the presence of outliers.

Djuric and Kay (1992) evaluated six different procedures belonging to the group of the penalty function methods. Only pure autoregressive processes were considered in the study. The evaluated methods showed relatively good accuracy even for short time series of 40 observations. For the first- and second-order autoregressive models, the percentage of correct selections varied between 59 and 97 . 
Increasing the sample size improved the performance of all procedures. Other factors affecting the quality of identifications were the degree of dependency (better results were obtained for large $\phi$ values) and parameterizations in higher order models (different results for narrow- and wideband processes were observed).

\section{Summary}

Model identification is one of the most challenging and distressing issues in ARMA modeling. Various automated methods for ARMA model identification have been developed during the last three decades to make the model identification process more reliable and less subjective. Three procedures, MINIC, SCAN, and ESACF, are freely available in current versions of SAS for Windows. Although numerous articles and books dealing with explanation of these procedures can be found in the time series literature, very few studies exist evaluating the methods. Therefore, the objectives of this article are to compare MINIC, SCAN, and ESACF as model identification tools for different ARMA structures and examine the influence of sample size, model complexity, and degree of dependency in a time series on their performance.

\section{METHOD}

The performance of identification methods was assessed on 11 ARMA models. Since most published studies in the behavioral sciences have identified stable series, only stationary processes were considered. We focused our Monte Carlo experiments on first- and second-order autoregressive and moving-average models because higherorder processes are tarely found in the social and behavioral sciences (Glass et al., 1975; Marsh \& Shibano, 1984; Rankin \& Marsh, 1985; Revenstorf, Kessler, Schindler, Hahlweg, \& Bluemner, 1980). In the first-order models, we used $\phi$ and $\theta$ values of $\pm 0.2, \pm 0.5$, and \pm 0.9 to represent different possible degrees of dependency. Parameter values of the second-order models ( 1.8 and -0.97 vs. 1.37 and -0.56) were employed in the evaluation study of $\mathrm{Dju}$ ric and Kay (1992). An ARMA (1, 1) model with $\phi=0.6$ and $\theta=-0.4$ was included to examine the advantages of pattern recognition methods ESACF and SCAN for mixed processes. The parameters $\phi$ and $\theta$ in the $\operatorname{ARMA}(1,1)$ model have opposite signs, so that the corresponding autoregressive and moving-average terms in the equation

$$
Y_{t}=\phi_{1} Y_{t-1}+U_{t}-\theta_{1} U_{t-1}
$$

do not approach parameter redundancy. For example, at $\phi=0.6, \theta=0.6$, cancellation would take place, and the apparent ARMA $(1,1)$ process would be in fact ARMA $(0,0)$. In cases of near-cancellation, the process may be well approximated by models with substantially different parameter values, leading to difficulty in evaluating the model identification methods. Such cases were ruled out by our design. The number of observations in simulated time series was varied between 40 and 200 . In time series literature (Box \& Pierce, 1970; Glass et al., 1975; Granger \& Newbold, 1986; Ljung \& Box, 1978; McCleary \& Hay, 1980; Velicer \& Fava, 2003) a minimum sample size of
50 observations is recommended as guideline. We used a minimum series length of 40 data points to compare our results with the study of Velicer and Harrop (1983). In sum, we manipulated the following variables: type of model, degree of dependency, and length of series. Each simulated model was replicated 1,000 times.

As a quality criterion, we computed the percentage of correct model selections. For detailed analysis, we also calculated the percentage of under- and overestimations of the model order. For SCAN and ESACF, only first recommendations in SAS output were considered. Recall that, in contrast to MINIC, SCAN, and ESACF can make several recommendations in each run. We regarded only the first ones for the following reasons. First, SAS orders recommendations of SCAN and ESACF by the number of insignificant terms contained in triangle or rectangular patterns, therefore the first suggestion is claimed to be the best one. Second, this is confirmed by our experience with more than 60,000 simulated series which revealed that in about $99 \%$ of the cases correct model identifications of SCAN and ESACF appear as the first recommendations in SAS output. In other words, if the first recommendation is false, the probability of selecting a correct model by means of subsequent recommendations is minute. Finally, considering only the first selections of SCAN and ESACF facilitates a straightforward comparison of their performance with that of MINIC, since a total number of possible identifications remains 1,000 for each method.

All computations and the generation of independent $N(0,1)$ innovations $U_{t}$ were performed with SAS System for Windows Version 9.1 software. In the IDENTIFY statement of PROC ARIMA, default SAS dimensions were used. The following SAS code gives ACF, PACF, and MINIC, SCAN, and ESACF recommendations.

$* * * * * * * * * * * * * * * * * * * * * * * * * * * * * * * * * * * *$
proc arima data= series;
identify var =y minic scan esacf;
run;
$* * * * * * * * * * * * * * * * * * * * * * * * * * * * * * * * * * * *$

\section{RESULTS}

Table 1 compares the accuracy of model selections of MINIC, SCAN, and ESACF in first-order processes. As can be seen, there are distinct differences in the performance of the evaluated methods. On average, MINIC did better in autoregressive than in moving-average cases. In contrast, ESACF yielded the highest percentage of correct selections for moving-average series. SCAN showed no clear preference referring to the type of model. Increase in number of observations had a much more pronounced effect for MINIC than for SCAN or ESACF. For all three methods, model identification was less accurate for low dependency than medium- or high-dependency models.

Figure 1 visualizes the accuracy of the identification procedures for different first-order structures. MINIC and SCAN outperformed ESACF in the autoregressive cases. The inferiority of ESACF is especially vivid for low- and medium-dependency parameterizations. The best result 
Table 1

Accuracy (Percentage Correct) of MINIC, SCAN, and ESACF for First-Order Models, Based on 1,000 Replications

\begin{tabular}{|c|c|c|c|c|c|c|c|c|c|c|}
\hline \multirow{3}{*}{$\begin{array}{l}\text { Type of } \\
\text { Model }\end{array}$} & \multirow[b]{3}{*}{$T$} & \multicolumn{9}{|c|}{ Degree of Dependency } \\
\hline & & \multicolumn{3}{|c|}{ Low (0.2) } & \multicolumn{3}{|c|}{ Medium (0.5) } & \multicolumn{3}{|c|}{ High (0.9) } \\
\hline & & MINIC & SCAN & $\overline{\text { ESACF }}$ & MNNIC & SCAN & $\overline{\text { ESACF }}$ & MINIC & SCAN & $\overline{\text { ESACF }}$ \\
\hline$(1,0)$ & $\begin{array}{l}40 \\
80 \\
100 \\
200\end{array}$ & $\begin{array}{l}10.4 \\
20.6 \\
23.9 \\
43.1\end{array}$ & $\begin{array}{l}15.6 \\
28.1 \\
33.3 \\
58.8\end{array}$ & $\begin{array}{l}8.9 \\
4.9 \\
6.1 \\
6.1\end{array}$ & $\begin{array}{l}38.9 \\
70.1 \\
74.4 \\
88.5\end{array}$ & $\begin{array}{l}59.1 \\
71.4 \\
74.5 \\
74.1\end{array}$ & $\begin{array}{l}37.9 \\
37.1 \\
35.2 \\
31.7\end{array}$ & $\begin{array}{l}54.6 \\
79.7 \\
83.6 \\
91.0\end{array}$ & $\begin{array}{l}69.7 \\
69.4 \\
70.3 \\
68.0\end{array}$ & $\begin{array}{l}70.6 \\
64.0 \\
64.0 \\
61.2\end{array}$ \\
\hline$(0,1)$ & $\begin{array}{l}40 \\
80 \\
100 \\
200\end{array}$ & $\begin{array}{l}11.6 \\
21.1 \\
25.6 \\
36.6\end{array}$ & $\begin{array}{r}10.2 \\
9.2 \\
9.4 \\
10.6\end{array}$ & $\begin{array}{l}11.0 \\
29.5 \\
38.2 \\
57.6\end{array}$ & $\begin{array}{l}24.3 \\
46.6 \\
52.1 \\
69.4\end{array}$ & $\begin{array}{l}28.9 \\
45.8 \\
50.2 \\
66.8\end{array}$ & $\begin{array}{l}45.3 \\
71.7 \\
75.1 \\
76.5\end{array}$ & $\begin{array}{l}25.9 \\
43.7 \\
49.3 \\
59.7\end{array}$ & $\begin{array}{l}64.1 \\
75.2 \\
76.4 \\
74.5\end{array}$ & $\begin{array}{l}76.5 \\
83.0 \\
81.5 \\
76.1\end{array}$ \\
\hline
\end{tabular}

(91.0) was achieved by MINIC for the $(1,0)$ model with $\phi=0.9$ and $T=200$. ESACF showed the worst result (4.9) for the $(1,0)$ model with $\phi=0.2$ and $T=80$. In the moving-average case, however, ESACF was superior to the other two methods regardless of sample size and degree of dependency. In time series with 200 observations, the percentage of correct identifications of ESACF was $57.6,76.5$, and 76.1 for low, medium, and high $\theta$ values, respectively. The SCAN method worked well in high- and medium-dependency situations (average $60 \%$ of correct selections). In the low-dependency case, however, its performance was disappointing with 10.6 percent of correct identifications at best. MINIC showed a reasonable performance only in large sample situations. For $T=200$, the percentages of correct identifications were 36.6 for $\theta=$ $0.2,69.4$ for $\theta=0.5$, and 59.7 for $\theta=0.9$.

Table 2 shows the percentage of correct selections for second-order and mixed models. For autoregressive processes, the effect of parameterization on the performance of the evaluated methods is apparent. SCAN and ESACF showed quite good results for the model with $\phi_{1}=1.8$ and $\phi_{2}=-0.97$. The accuracy rate was about $70 \%$, regardless of sample size. For the $(2,0)$ model with $\phi_{1}=1.37$ and $\phi_{2}=-0.56$, the quality of identifications of both procedures depended on time series length. Increasing the number of observations from 40 to 200 improved the performance of SCAN from $38.9 \%$ to $74 \%$. For ESACF however, increase in sample size reduced the percentage of correct identifications from $51.8 \%$ to $27 \%$. In contrast to SCAN and ESACF, the MINIC approach provided similar results for both $(2,0)$ models. In larger samples, MINIC distinctly outperformed the two other methods with more than $80 \%$ of correct selections.

Figure 2 visualizes the effect of parameterization for second-order models. Note that no effect of parameterization was observed in the moving-average case: Similar results were obtained for both $(0,2)$ models. Figure 3 explains these findings. Changing parameter values altered spectral characteristics of the autoregressive processes. The spectral density function ${ }^{1}$ of ARMA $(2,0)$ model with $\phi_{1}=1.8$ and $\phi_{2}=-0.97$ demonstrates that variance of this series concentrates at one frequency. For the $(2,0)$ process with $\phi_{1}=1.37 \phi_{2}=-0.56$, however, there are two dominant cycles. In contrast, both moving-average series show the same broadband spectral pattern despite of different parameterizations.

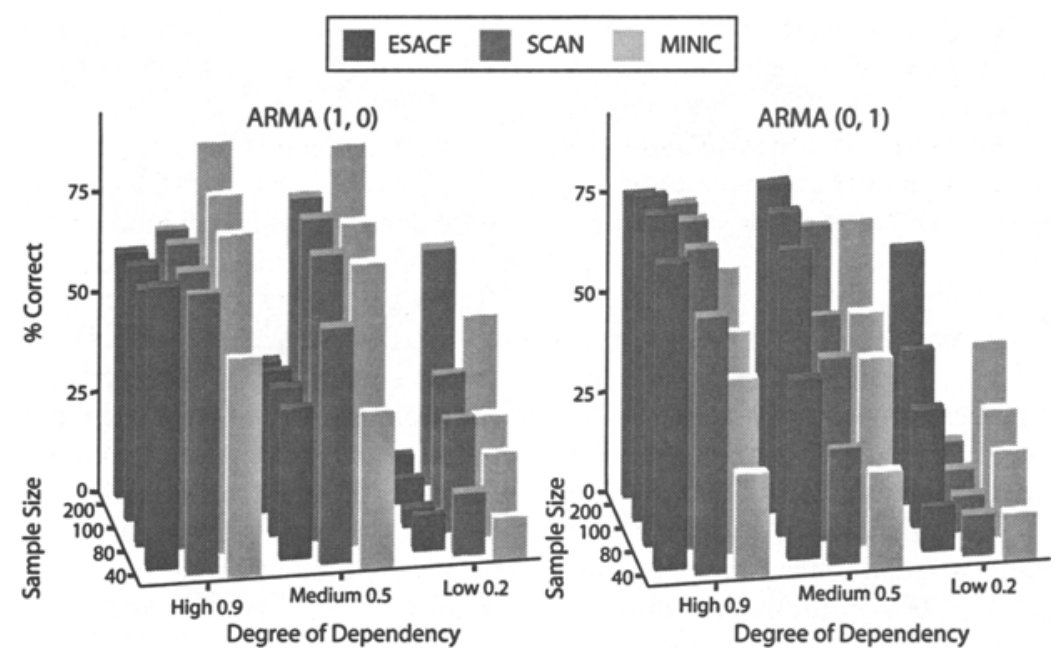

Figure 1. Comparison results (percentage correct) for first-order models, based on 1,000 replications. 
Table 2

Comparison Results (Percentage Correct) for Second-Order and Mixed Models, Based on 1,000 Replications

\begin{tabular}{llrrrr} 
& & \multicolumn{5}{c}{ Number of Observations } \\
\cline { 3 - 6 } \multicolumn{1}{c}{ Type of Model } & Method & $T=40$ & $T=80$ & $T=100$ & $T=200$ \\
\hline$(2,0)$ & MINIC & 52.0 & 78.0 & 81.3 & 90.4 \\
$\phi_{1}=1.8, \phi_{2}=-0.97$ & SCAN & 70.5 & 71.5 & 72.7 & 73.1 \\
& ESACF & 72.5 & 72.9 & 72.4 & 70.7 \\
$(2,0)$ & MINIC & 48.6 & 78.2 & 82.0 & 91.7 \\
$\phi_{1}=1.37, \phi_{2}=-0.56$ & SCAN & 38.9 & 58.7 & 66.1 & 74.0 \\
& ESACF & 51.8 & 40.1 & 36.4 & 27.0 \\
$(0,2)$ & MINIC & 3.0 & 2.4 & 3.2 & 6.1 \\
$\theta_{1}=1.8, \theta_{2}=-0.97$ & SCAN & 9.5 & 9.7 & 12.0 & 13.1 \\
& ESACF & 8.1 & 37.9 & 54.1 & 72.8 \\
$(0,2)$ & MINIC & 2.9 & 6.5 & 7.0 & 16.1 \\
$\theta_{1}=1.37, \theta_{2}=-0.56$ & SCAN & 5.8 & 7.8 & 9.6 & 10.1 \\
& ESACF & 4.4 & 26.1 & 40.6 & 66.2 \\
$(1,1)$ & MINIC & 3.9 & 19.6 & 25.9 & 45.4 \\
$\phi=0.6, \theta=-0.4$ & SCAN & 13.3 & 49.4 & 64.2 & 79.1 \\
& ESACF & 6.4 & 31.2 & 40.4 & 44.3 \\
\hline
\end{tabular}

As can be seen from the bottom section of Table 2, in the mixed $(1,1)$ case SCAN showed the best results followed by ESACF and MINIC. Increase in number of observations improved the performance of all three approaches.

Figures 4-6 present the frequency distribution of identified patterns. In addition to correct identifications, the figures depict the percentage of under- and overestimations of the model order and selections of different model structure. For instance, selecting models with $p>1$ for simulated $(1,0)$ series means overestimation of the autoregressive order. In the autoregressive case, moving-average and mixed models represent alternative structures to the true one.

As Figure 4 illustrates, the performance of MINIC and SCAN for first-order autoregressive models with low degree of dependency appears to be very similar. The number of correct selections increased with time series length. For sample sizes of less than 200 observations, both procedures logically selected the $(0,0)$ process about $50 \%$ of the time to describe the behavior of the data. Despite an extreme low percentage of correct identifications, ESACF concluded in most cases that either $(0,0)$ or $(0,1)$ models could also have generated the data (which makes sense, since first-order autoregressive and moving-average processes with low-valued parameterizations have spectral characteristics nearly equivalent to those of white noise). As compared with MINIC and SCAN, the ESACF approach selected a distinctly larger percentage of higher order mixed ARMA structures. For example, the AR (1) model with $\phi=0.2$ was identified as mixed in $17.8 \%$ of the trials in series with $T=200$. The tendency of ESACF to overparameterization was even more pronounced for structures with medium and large parameter values. In sum, MINIC showed the best performance in the $(1,0)$ case. In series with medium and high degrees of dependency, the number of incorrect selections practically vanished with increasing sample size.

As can be seen from Figure 5, ESACF was superior to the other two methods in first-order moving-average cases. Depending on parameter values most series (about $70 \%$ ) were either correctly classified or identified as parsimoniously near equivalent mathematical structures. Analysis of incorrect selections revealed that ESACF and SCAN showed similar tendency to favor higher-order mixed models (10\%-20\%). Overparameterization represented the most frequent error of MINIC for patterns with $\theta=0.9$ : most series were identified as higher-order mixed or pure structures. In models with low and medium degrees of dependency, MINIC classified incorrectly about $20 \%$ of trails as ARMA $(1,0)$.

Figure 6 shows the frequency distribution of model selections for second-order and mixed processes. As the upper section of Figure 6 illustrates, the evaluated methods almost never underestimated the order of $(2,0)$ series. MINIC tended to overestimate the model order. For SCAN and ESACF, the most frequent incorrect selections were higher-order mixed models. It is apparent from the middle section of Figure 6 that MINIC and SCAN were seldom able correctly to identify simulated $(0,2)$ structures. SCAN

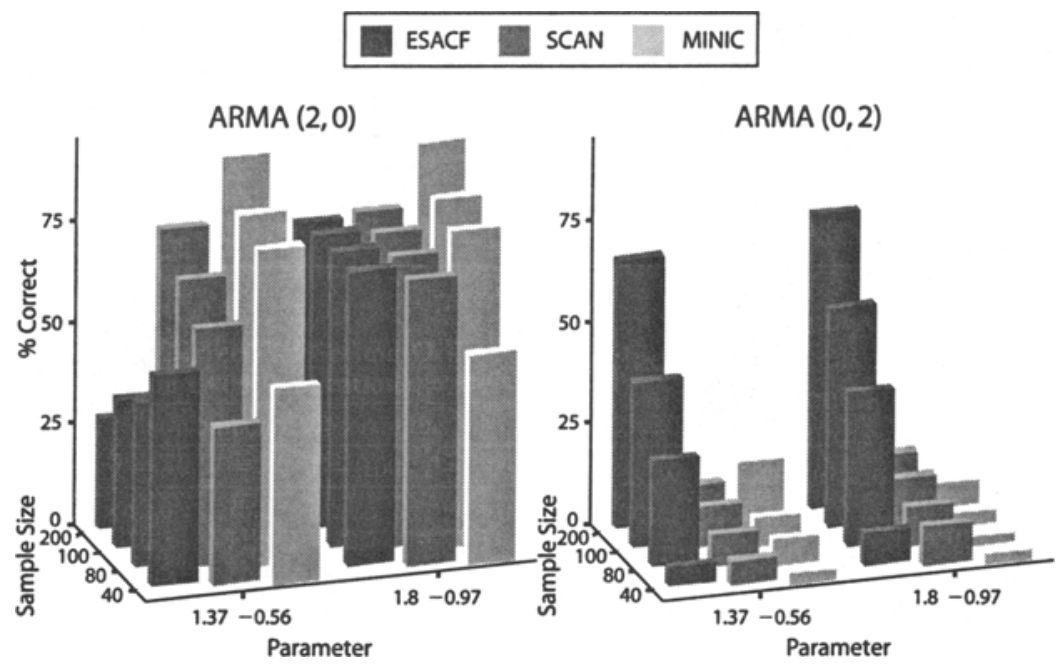

Figure 2. Comparison results (percentage correct) for second-order models, based on 1,000 replications. 

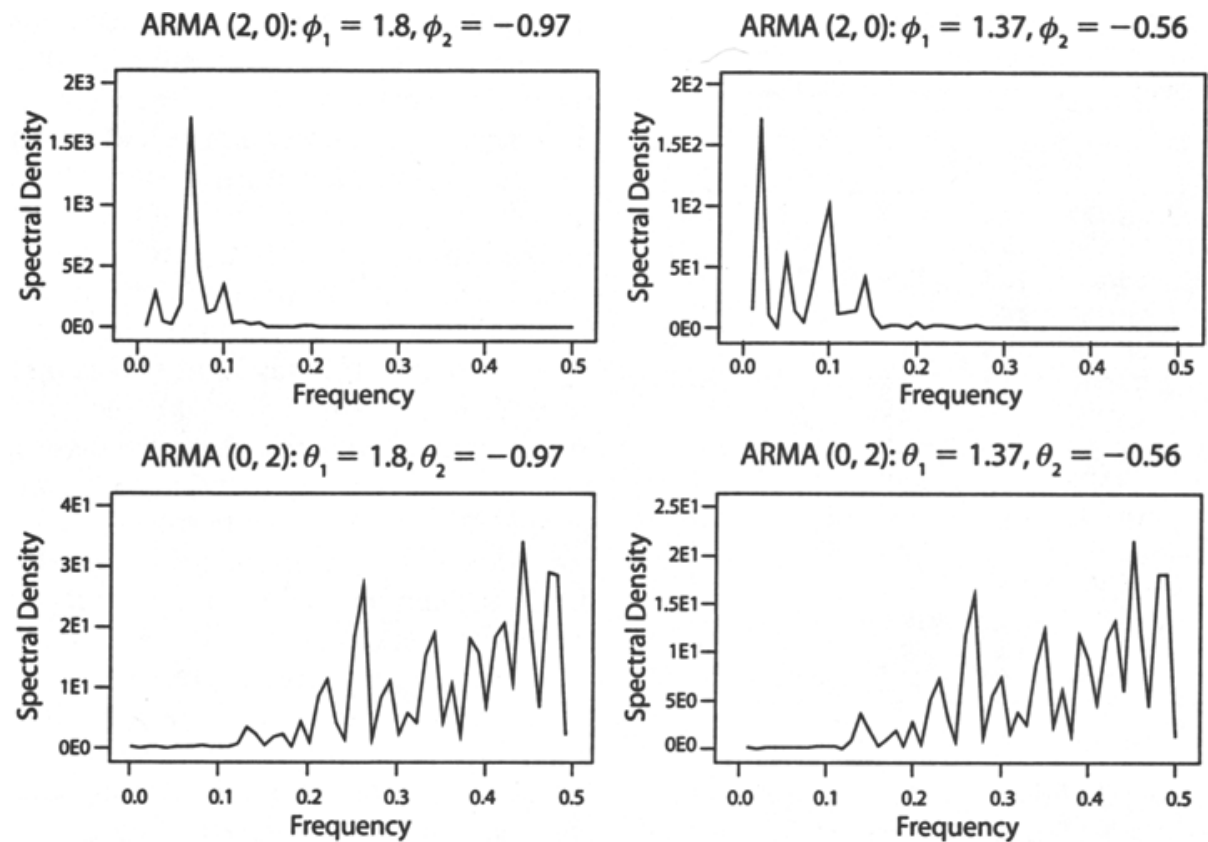

Figure 3. Spectral density functions of simulated second-order processes with $t=100$ (distribution of the time series variance across different frequencies $[f], f=1 / \tau$, with $\tau=$ length of a cycle).

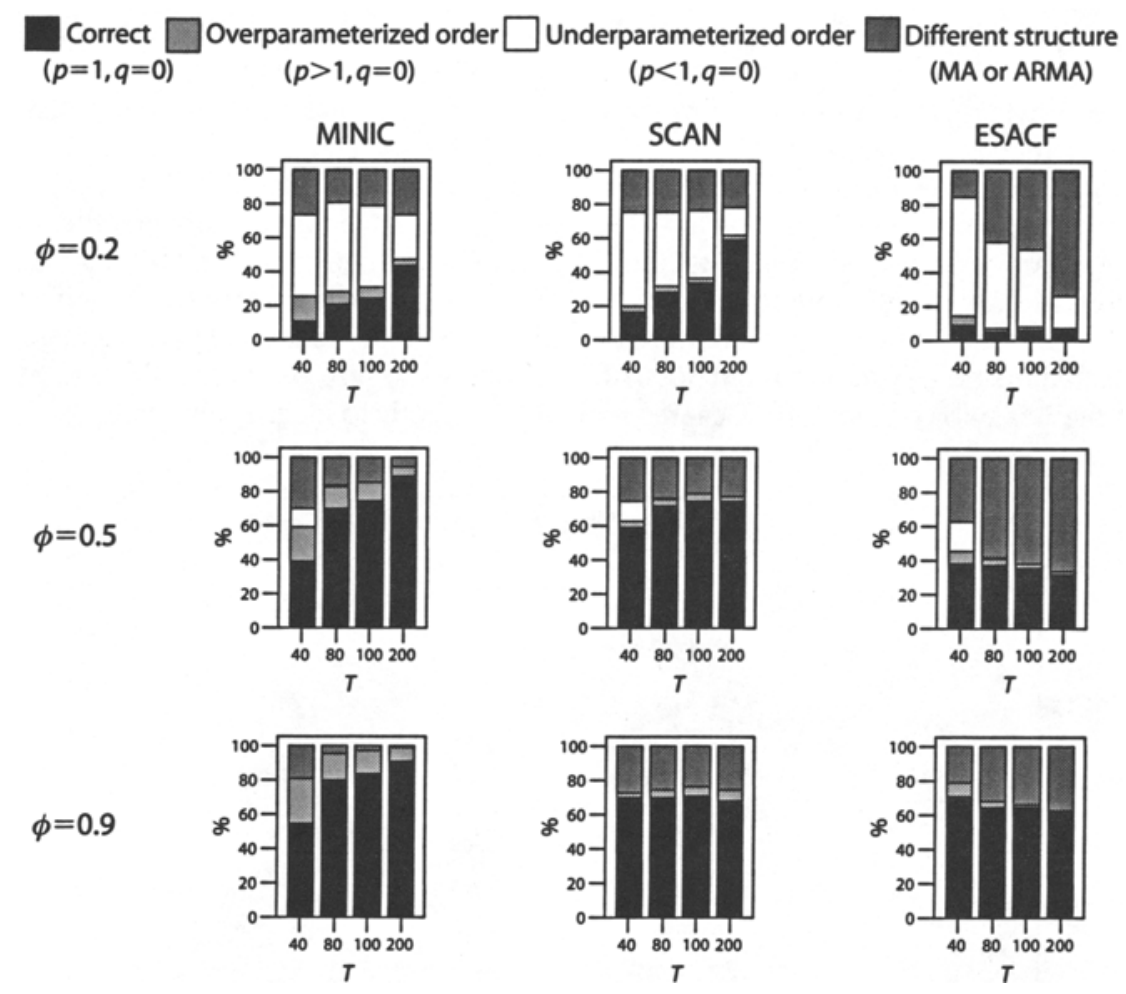

Figure 4. Frequency distribution of identified structures for $(1,0)$ models, recelved from 1,000 replications. 
Correct $\square$ Overparameterized order $\square$ Underparameterized order $\square$ Different structure $\begin{array}{lll}(p=0, q=1) & (p=0, q>1) & (p=0, q<1)\end{array} \quad$ (AR or ARMA)

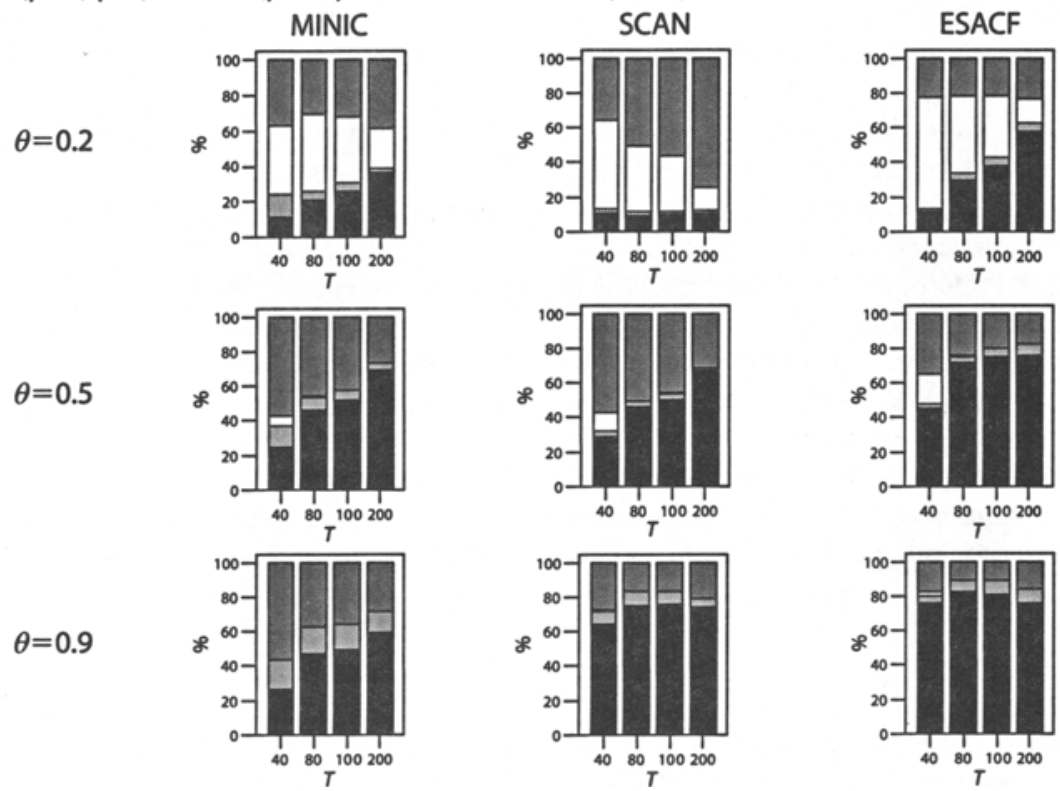

Figure 5. Frequency distribution of identified structures for $(0,1)$ models, received from 1,000 replications.

Correct $\square$ Overparameterized order $\square$ Underparameterized order $\square$ Different structure $(p=2, q=0) \quad(p<2, q=0)$ (MA or ARMA)

Model

$(2,0)$

$\phi_{1}=1.37$

$\phi_{2}=-0.56$
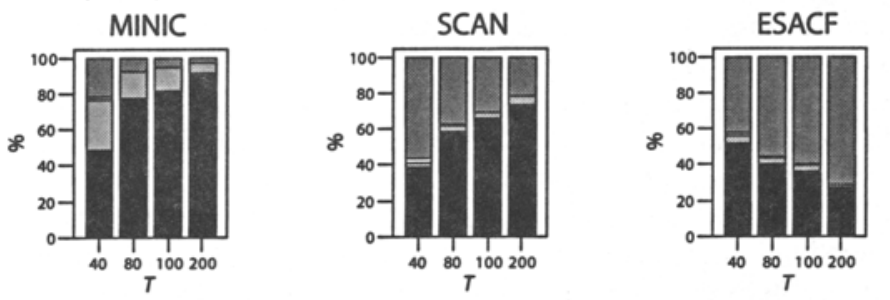

Correct $\square$ Overparameterized order $\square$ Underparameterized order $\square$ Different structure $\begin{array}{llll}(p=0, q=2) & (p=0, q>2) & (p=0, q<2) & \text { (AR or ARMA) }\end{array}$

Model
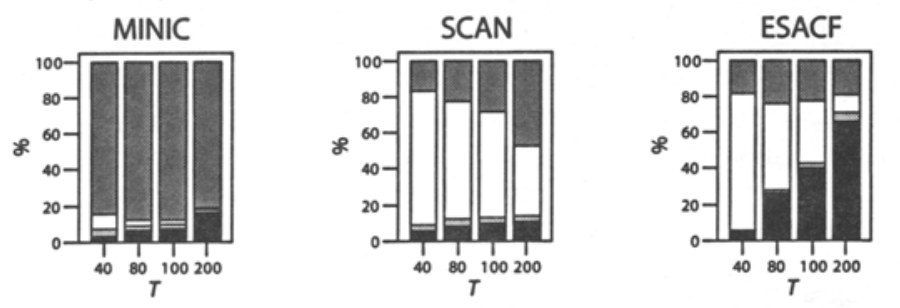

$(0,2)$

$\theta_{1}=1.37$

$\theta_{2}=-0.56$

Correct $\square$ AR Models $\square$ MA Models $\square$ Higher order mixed models

Model
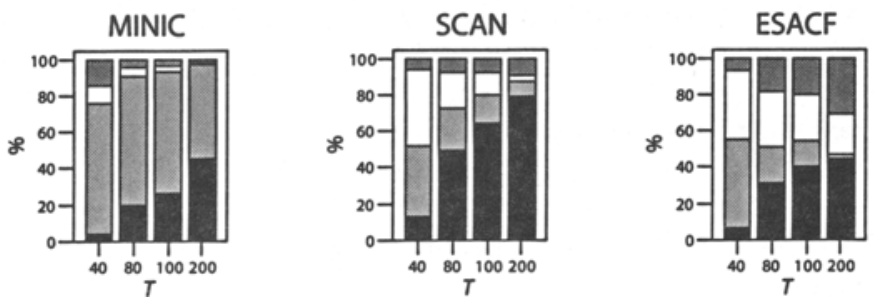

Figure 6. Frequency distribution of identified structures for $(2,0),(0,2)$, and $(1,1)$ ARMA models, received from 1,000 replications. 
either underestimated the model order or misclassified moving-average series as mixed patterns. MINIC misidentified most $(0,2)$ series as mixed processes. ESACF outperformed the two other methods. Increasing the sample size visibly refined the accuracy of the ESACF approach. In smaller samples however, a strong tendency to underestimate the model order was observed. The bottom section of Figure 6 visualizes that in the mixed case the performance of all three approaches was dependent on sample size. The SCAN method was superior to both MINIC and ESACF. In smaller samples, SCAN selected either autoregressive or moving-average structures of first-order as identification alternatives to the $(1,1)$ model. MINIC incorrectly classified about $40 \%$ of trials as $(2,0)$ structures. ESACF misclassified most series as either moving-average structures or mixed models with $p$ and $q>1$. For $T=40$, however, the most frequent incorrect selections of ESACF in the mixed case were autoregressive models (46.6\%).

\section{DISCUSSION}

In this article we empirically investigated the accuracy of three automated procedures for ARMA model identification commonly available in current versions of SAS for Windows. Unfortunately, our simulation results do not allow announcing the universally best approach. For autoregressive structures, MINIC achieved the best results. SCAN was superior to the other two procedures for mixed $(1,1)$ models. For moving-average processes, ESACF obtained the most correct selections. MINIC and SCAN had difficulty identifying moving-average models. ESACF demonstrated low power in autoregressive cases. For all three methods, model identification was less accurate for low dependency than medium- or high-dependency models. SCAN and ESACF tended to select higher-order mixed structures, especially in larger samples.

The results of the present study are consistent with findings reported in the literature. Congruent with the conclusions of Dickey (2004), SCAN was the most accurate method followed by ESACF and MINIC in the mixed $(1,1)$ case. Accordant with the findings of Koreisha and Yoshimoto (1991), we observed the tendency of ESACF to overparameterization, its difficulty with autoregressive models, better accuracy for larger parameter values in pure cases, and insensitivity to sample size changes. The only difference refers to the performance of ESACF for $(0,1)$ models. Koreisha and Yoshimoto (1991) reported maximally $60 \%$ of correct selections. The best result in the present study was $83 \%$ for $\theta=0.9$ and $T=80$. For processes with medium and high degrees of dependency, the percentage of correct identifications was about $70 \%$, regardless of sample size. Djuric and Kay (1992) demonstrated that the accuracy of some methods depended on spectral characteristics of underlying processes. The same phenomenon was observed in the present study. SCAN and ESACF yielded significantly better results in narrowband case than in broadband. The performance of MINIC appeared to be independent of spectral properties of simulated $(2,0)$ series.

Table 3 compares the accuracy of automated methods with the results reported by Velicer and Harrop (1983) for highly trained judges. For the comparison, only similar models and parameterizations were considered. With the average at $60 \%$ of correct selection all three methods were distinctly superior to subjective identifications for autoregressive series. The superiority of automated methods was especially pronounced in the $(2,0)$ case. In the moving-average case, however, only ESACF outperformed trained judges. SCAN worked about equally well. The performance of MINIC was distinctly below that of the judges, especially for $T=40$.

For better understanding of the reported findings, it is important to bear in mind that the percentage of correct model selections is not the only quality criterion we can use to evaluate the procedures. Note that despite the lack of correct identifications in low-dependency models with a small number of observations, performance of the automated methods can be classified as satisfactory, because the most alternatives to the true models were parsimonious nearly equivalent mathematical representations. For instance, selecting the $(0,0)$ model for first-order autoregressive or moving-average processes with low degrees of dependency is entirely plausible in smaller samples, since spectral characteristics of these structures are nearly equivalent to those of white noise. For the same reason, Koreisha and Yoshimoto attested the ARCRI approach an excellent performance for the $(\phi=0.8, \theta=0.5)$ parameterization notwithstanding the fact that the percentage of correct identifications was 0.0 for $T=50,1.0$ for $T=100$, and 5.0 for $T=200$ (Koreisha \& Yoshimoto, 1991, pp. 46 and 50). Based on this less rigorous criterion, for the majority of structures studied, at least $60 \%$ of model selections of MINIC, SCAN, and ESACF can be classified as correct. This result, however, is still markedly below that of the ARCRI procedure $(80 \%-100 \%$ of correct selections for series between 100 and 200 observations).

The ARCRI approach, also termed the residual white noise autoregressive criterion (RWNAR), proposed by Pukkila, Koreisha, and Kallinen (1990), consists in systematically fitting increasing-order $\operatorname{ARMA}(p, q)$ structures to the data employing linear estimation procedures, and then checking the randomness of the residuals. This iterative process is terminated, when the innovations obtained from the fitted model are white noise. A two-stage regression technique of $A R C R I$ is fairly similar to the MINIC algorithm. However, ARCRI is based on general-

Table 3

Comparison of Correct Model Identifications With Results of Velicer and Harrop (1983)

\begin{tabular}{cccccc}
\hline \multirow{2}{*}{$\begin{array}{c}\text { Type of } \\
\text { Model }\end{array}$} & $T$ & $\begin{array}{c}\text { Subjective } \\
\text { Judgments }\end{array}$ & \multicolumn{3}{c}{ Automated Methods* } \\
\cline { 4 - 6 }$(1,0)$ & 40 & 19 & 47 & 64 & 54 \\
& 100 & 46 & 79 & 72 & 50 \\
$(0,1)$ & 40 & 46 & 25 & 46 & 61 \\
& 100 & 67 & 51 & 63 & 78 \\
$(2,0)$ & 40 & 0 & 50 & 55 & 62 \\
& 100 & 4 & 82 & 69 & 54 \\
$M$ & & 30 & 56 & 51 & 60 \\
\hline
\end{tabular}

"In first-order models, only medium and high degrees of dependency are considered. 
ized least squares and differs in the penalty function. Unfortunately, the ARCRI method is not available in popular statistics packages. Surely, it can be implemented on any computer system with a linear regression program. The necessary programming adjustment, however, could be rather difficult for applied researchers. Moreover, to our knowledge, no studies exist replicating the remarkable findings of Koreisha and Yoshimoto (1991).

An important aim of the present study was to examine the influence of sample size and parameter values on performance of the evaluated methods. The effect of sample size was more pronounced for MINIC than for SCAN and ESACF. Increased number of observations refined the performance of MINIC. For SCAN and ESACF however, in some cases increasing sample size reduced the percentage of correctly. identified structures. This decrease was stronger for ESACF than for SCAN. Koreisha and Yoshimoto (1991) reported a similar tendency of ESACF. For some simulated structures, rather strong effects emerge. In the ARMA $(1,1)$ case, for instance, the decrease in percentage of correct identifications changed from $58 \%$ with $T=50$ to $13 \%$ with $T=1,000$ (Koreisha \& Yoshimoto, 1991, p. 51). This effect is probably due to underestimation of the variance of extended sample autocorrelations because of the employed approximation (Tsay $\&$ Tiao, 1984, p. 87). To explore this issue, Koreisha and Yoshimoto manipulated the confidence level of ESACF and showed that for samples with $T>500$ using \pm 2 standard errors $(s)$ is inappropriate and leads to identification of very high-order models. For sample sizes between 100 and 200 observations, increasing the confidence interval to $\pm 3 s$ substantially improves the performance of ESACF. For smaller samples, no clear effect was observed (Koreisha \& Yoshimoto, 1991, pp. 51-53).

The failure of SCAN and ESACF to refine their identification with increasing $T$ represents a clear drawback of these methods. As noted previously, SCAN and ESACF exhibit the tendency to select higher-order mixed models in larger samples. Thus, for long time series the ESACF or SCAN tables provide information only on the maximum values of $p$ and $q$ (Tsay \& Tiao, 1984, p. 95).

The effect of parameterizations is unequivocal for first-order processes. For all three methods, the quality of selections was less accurate for low dependency than medium- or high-dependency models. Recall that the best result $(91.0)$ was achieved by MINIC for the $(1,0)$ model with $\phi=0.9$. The ability of automated methods to recognize autoregressive structures with large parameter values (with roots close to the unit circle) could be used in applied settings for discrimination of time series on the edge of instationarity from their nonstationary alternatives. The point is that autocorrelation and partial autocorrelation functions of the ARIMA $(0,1,0)$ represented by the equation

$$
Y_{t}=Y_{t-1}+U_{t}
$$

are scarcely distinguishable from those of the ARIMA $(1,0,0)$ represented by the equation

$$
Y_{t}=0.9 Y_{t-1}+U_{t} \text {. }
$$

Despite a striking similarity of their ACFs and PACFs, however, both models possess quite different characteristics. For instance, the ARIMA $(0,1,0)$, also called random walk or brown noise, have an infinite memory. In contrast, the memory of the ARIMA $(1,0,0)$ is short implying that this process is predictable only from its immediate past. Random walk is a popular model increasingly employed for explanation of various psychological and behavioral phenomena (Gilden, 2001; Marks-Tarlow, 1999; Thornton \& Gilden, 2005; Wagenmakers et al., 2004). Thus for applied researchers, it is important to distinguish it from stationary autoregressive processes with $\phi$ near 1 . Even special procedures called unit root tests developed to test stationarity conditions show an extremely low power in this case.

For higher-order structures, the effect of parameterization is much more difficult to generalize. The results for the $(0,2)$ models showed that different parameter values could imply the same spectral pattern. For mixed series, possible cancellation effects for autoregressive and moving-average terms with the same signs might seriously affect the accuracy of identification, since models with a smaller number of parameters represent an adequate fit for those data. The higher the degree of complexity the more restricted are any generalizations. For instance, Chan (1999) observed for the $(2,1)$ model with the $(1.3,-0.4$, $-0.5)$ parameterization that the quality of model identifications of ESACF became more exact with increasing number of observations. The percentage of correct selections visibly improved from 13.0 for $T=100$ to 33.5 for $T=1,000$. In the study of Koreisha and Yoshimoto (1991), however, the accuracy of ESACF for the ARMA $(2,1)$ with similar parameter values $(1.4,-0.6,-0.8)$ became distinctly worse in larger samples. The percentage of correct selections was $45,35,21$, and 10 for $T=100$, 200,500 , and 1,000 , respectively. On the other hand, no effect of sample size was found in the mixed $(2,1)$ case with the $(-0.5,-0.9,0.6)$ parameterization: the percentage of correct identifications was about $60 \%$, regardless of time series length (Koreisha \& Yoshimoto, 1991, p. 53). According to Chan (1999), ESACF outperformed SCAN for the $(2,1)$ model with $\phi_{1}=1.3, \phi_{2}=-0.4, \theta=-0.5$. We failed to replicate this result. Table 4 shows that for different simulated mixed $(2,1)$ structures SCAN were always superior to ESACF. In our study, however, only first recommendations of SCAN and ESACF in SAS output were considered. (The total number of identifications was always 1,000 .)

Recall that both procedures can make several recommendations in each run. In contrast to our simulations, Chan (1999) employed as a quality criterion the identification ratio considering multiple identifications. We think that using the identification ratio

$$
R=\frac{\text { Number of correct identifications in } 1,000 \text { runs }}{\text { Total number of identifications in } 1,000 \text { runs }}
$$

may produce misleading results for the following reasons. According to our experience with simulated series, correct model identifications of SCAN and ESACF constantly 
Table 4

Comparison Results (Percentage Correct) for $(2,1)$ Mixed Models, Based on 1,000 Replications

\begin{tabular}{clccccc}
\hline & & \multicolumn{5}{c}{ Number of Observations } \\
\cline { 3 - 7 }$\left(\phi_{1}, \phi_{2}, \theta_{1}\right)$ & Method & $T=40$ & $T=100$ & $T=200$ & $T=400$ & $T=1,000$ \\
\hline$(1.3,-0.4,-0.5)$ & MINIC & 2.2 & 13.2 & 37.5 & 63.1 & 82.8 \\
& SCAN & 2.8 & 38.4 & 69.2 & 82.1 & 80.5 \\
& ESACF & 1.9 & 21.5 & 26.9 & 21.6 & 17.5 \\
$(1.4,-0.6,-0.8)$ & MINIC & 6.4 & 19.7 & 27.4 & 32.4 & 40.0 \\
& SCAN & 19.2 & 66.9 & 80.4 & 81.3 & 80.2 \\
& ESACF & 20.0 & 49.8 & 38.8 & 34.5 & 31.5 \\
$(1.8,-0.9,-0.5)$ & MINIC & 2.6 & 8.7 & 14.1 & 22.3 & 32.0 \\
& SCAN & 51.0 & 78.0 & 79.8 & 79.8 & 79.0 \\
& ESACF & 36.0 & 68.6 & 68.8 & 68.6 & 69.0 \\
$(-0.5,-0.9,0.6)$ & MINIC & 10.3 & 40.8 & 59.3 & 72.7 & 86.0 \\
& SCAN & 41.7 & 73.2 & 80.1 & 80.0 & 79.7 \\
& ESACF & 36.6 & 60.3 & 53.6 & 51.4 & 50.4 \\
\hline
\end{tabular}

appear as the first recommendations in SAS output. Chan demonstrated that, relative to ESACF, SCAN generated a distinctly larger quantity of model identifications. For instance, the numbers of selected combinations in series with 100 observations were 2,634 for ESACF and 2,982 for SCAN (Chan, 1999, p. 75). Therefore, employing the identification ratio as a quality criterion is especially disadvantageous for SCAN. Suppose that all evaluated methods would perform equally well with 500 correct model selections in 1,000 runs. The total numbers of possible identifications were, however, 1,000 for MINIC, 2,634 for ESACF, and 2,982 for SCAN. Thus we obtain quite different identification ratios: 0.5 for MINIC, 0.19 for ESACF, and 0.17 for SCAN.

Table 5 containing a typical SAS output of the IDENTIFY statement of PROC ARIMA illustrates that multiple recommendations of SCAN and ESACF usually include models of different type. Thus for applied researchers, it is important to know that only the first recommendations are useful.

Unfortunately, none of the evaluated procedures is able to identify seasonal structures or applicable to transfer function models. In contrast to MINIC, ESACF, and SCAN can handle directly nonstationary series. For integrated processes, this means that both methods can be applied to the original nondifferenced data.

\section{Concluding Remarks}

The general conclusions from the present study are (1) in the pure structures, MINIC and SCAN perform well for autoregressive models, whereas ESACF works better in

Table 5

Recommendations of MINIC, SCAN, and ESACF for an Empirical Series

Minimum Table Value: $\operatorname{BIC}(1,0)=-2.4567$

ARMA $(p+d, q)$ Tentative Order Selection Tests

\begin{tabular}{cccccc} 
p+d & $q$ & BIC & p+d & $q$ & BIC \\
1 & 1 & -2.45065 & 2 & 0 & -2.45657 \\
2 & 0 & -2.45657 & 1 & 1 & -2.45065 \\
0 & 5 & -2.3537 & 0 & 5 & -2.3537 \\
\hline
\end{tabular}

moving-average cases; (2) model identifications are more precise for high dependency processes; (3) SCAN and ESACF are superior to MINIC for mixed $(1,1)$ models; (4) the positive effect of sample size is more pronounced for MINIC than for SCAN and ESACF; (5) SCAN and ESACF tend to select higher-order mixed structures in larger samples. These conclusions are confined to stationary nonseasonal time series.

The reported findings of our Monte Carlo experiments could help in choosing an appropriate identification procedure if some knowledge about properties of the stochastic process under study is available. In economics and engineering sciences, for example, mixed models predominate (see Granger \& Newbold, 1986, for explanations). Physiological processes such as heart rate or brain activity are often autoregressive. In social and behavioral sciences, the most widespread processes are autoregressive and moving average of first or second order (Glass et al., 1975; Marsh \& Shibano, 1984; Revenstorf et al., 1980).

Although the evaluated methods were superior to subjective judgments, for some models and parameterizations their accuracy remained disappointing. Moreover, precise model identification is not guaranteed, even in very large samples. In the majority of applied settings researchers are unlikely to know the type of ARIMA process underlying their data, so it is precarious to rely on a particular procedure. Therefore, for applied researchers employing MINIC, SCAN, and ESACF, our findings strongly support the recommendation of Box et al. (1994) to use automated methods as supplementary guidelines in the model selection process and not as a substitute for critical examination of the ACF, PACF and model residuals. In other words, only an elaborated strategy combining different methods could ensure accurate model selection. Based on the results of the present study, Stadnytska, Braun, and Werner (2007) developed a methodology for model selection combining objective and subjective techniques and demonstrated its usefulness on empirical data.

\section{AUTHOR NOTE}

The authors are grateful to the anonymous reviewers for their helpful suggestions. Correspondence relating to this article may be sent to 
J. Werner, Department of Psychology, University of Heidelberg, Hauptstrasse 47-51, 69117 Heidelberg, Germany (e-mail: joachim.werner@ psychologie.uni-heidelberg.de).

\section{REFERENCES}

AKAIKE, H. (1974). A new look at the statistical model identification. IEEE Transactions on Automatic Control, 19, 716-723.

Algina, J., \& SWaminathan, H. A. (1977). A procedure for the analysis of time series designs. Journal of Experimental Education, 45, 56-60.

Algina, J., \& Swaminathan, H. A. (1979). Alternatives to Simonton's analysis of interrupted and multiple-group time series designs. Psychological Bulletin, 86, 919-926.

Beguin, J. M., Gourieroux, C., \& Montrort, A. (1980). Identification of mixed autoregressive-moving average process: The corner method. In O. D. Anderson \& M. R. Perryman (Eds.), Time series analysis: Proceedings of the international conference held at Houston, Texas, August 1980 (pp. 423-436). Amsterdam: North-Holland.

BoWERMAN, B. L., \& O'CoNNELL, R. T. (1993). Forecasting and time series: An applied approach. Belmont: Duxbury Press.

Box, G. E. P., \& JEnkins, G. M. (1970). Time series analysis: Forecasting and control. San Francisco: Holden-Day.

Box, G. E. P., Jenkins, G. M., \& ReInsel G. C. (1994). Time series analysis: Forecasting and control (3rd ed.). Englewood Cliffs, NJ: Prentice Hall.

Box, G. E. P., \& Pierce, W. A. (1970). Distribution of residual autocorrelations in autoregressive-integrated moving average time series models. Journal of American Statistical Association, 65, 1509-1526.

BROCKWELL, P. J., \& DAVIS, R. A. (2002). Introduction to time series and forecasting (2nd ed.). New York: Springer.

CHAN, W.-S. (1999). A comparison of some of pattern identification methods for order determination of mixed ARMA models. Statistics \& Probability Letters, 42, 69-79.

CHоl, В. S. (1992). ARMA model identification. New York: Springer.

CROSBIE, J. (1993). Interrupted time-series analysis with brief singlesubject data. Journal of Consulting \& Clinical Psychology, 61, 966-974.

Delcor, L., Cadopi, M., DFlignières, D., \& Mesure, S. (2002). Dynamics of the memorization of a morphokinetic movement sequence. Neuroscience Letters, 336, 25-28.

Dick一y, D. A. (2004). Applied time series notes (Notes). Available at www.stat.ncsu.edu/people/dickey/st730/Notes/p._38_55.pdf.

DJURIC, P. M., \& KAY, S. M. (1992). Order Selection of autoregressive models. IEEE Transactions on Acoustics, Speech, \& Signal Processing, 40, 2829-2833.

ForTES, M., Ninot, G., \& Delignières, D. (2005). The auto-regressive integrated moving average procedures implications for adapted physical activity research. Adapted Physical Activity Quarterly, 22, 221-236.

Fraschini E. M., \& AXAUSTEN, K. W. (2001). Day on day dependencies in travel: First results using ARIMA modeling (Working paper). Available at e-collection.ethbib.ch.

GILDEN, D. L. (2001). Cognitive emissions of $1 / f$ noise. Psychological Review, 108, 33-56.

Glass, G. V., Willson, V. L., \& Gottman, J. M. (1975). Design and analysis of time-series experiments. Boulder, CO: Colorado Associated University Press.

Gotrman, J. M. (1981). Time-series analysis. Cambridge: Cambridge University Press.

Granger, C. W. J., \& NeWBOLD, P. (1986). Forecasting economic time series. San Diego: Academic Press.

HanNAN, E. J., \& Rissanen, J. (1982). Recursive estimation of mixed autoregressive moving average order. Biometrika, 69, 81-94.

HARROP, J. W., \& VELICER, W. F. (1985). A comparison of three alternative methods of time series model identification. Multivariate Behavioral Research, 20, $27-44$.

HARROP, J. W., \& VELICER, W. F. (1990). Computer programs for interrupted time series analysis: II. A quantitative evaluation. Multivariate Behavional Research, 25, 233-248.
HuITEMA, B. E. (2002). Time-series intervention anabysis using ITSACORR: Fatal flaws. Manuscript submitted for publication.

HuITEMA, B. E. (2004). Analysis of interrupted time-series experiments using ITSE: A critique. Understanding Statistics, 3, 27-46.

Koreisha, S. G., \& PUKKILA, T. M. (1990). A generalized least-squares approach for estimation of autoregressive moving-average models. Journal of Time Series Analysis, 11, 139-151.

Koreisha, S. G., \& Yoshmoto, G. (1991). A comparison among identification procedures for autoregressive moving average models. International Statistical Review, 59, 37-57.

LuUNG, G. M., \& Box, G. E. P. (1978). On a measure of lack of fit in time series models. Biometrika, 65, 297-303.

MaKRIDAKIS, S., WheELWRIGHT, S. C., \& HyNDMAN, R. J. (1998). Fonecasting: Methods and applications. New York: Wiley.

MARKS-TARLOW, T. (1999). The self as a dynamical system. Nonlinear Dynamics, Psychology \& Life Sciences, 3, 311-345.

MARSH, J. C., \& Shibano, M. (1984). Issues in the statistical analysis of clinical time-series data. Social Work Research \& Abstracts, 20, 7-12.

MCCleARY, R., \& HAY, R. A., JR. (1980). Applied time series analysis for the social sciences. Beverly Hills, CA: Sage.

McKnight, S., McKean, J. W., \& Hurtema, B. E. (2000). A double bootstrap method to analyze linear models with autoregressive error terms. Psychological Methods, 5, 87-101.

Ninot, G., ForTes, M., \& Delignières, D. (2005). The dynamics of self-esteem in adults over a six-month period: An exploratory study. Journal of Psychology, 139, 315-330.

Pukkila, T. M., Koreisha, S., \& Kallinen, A. (1990). The identification of ARMA models. Biometrika, 77, 537-548.

RANKIN, E. D., \& MARSH, J. C. (1985). Effects of missing data on the statistical analysis of clinical time series. Social Work Research \& Abstracts, 21, 13-16.

Revenstorf, D., Kessler, A., Schindler, L., Hahlweg, K., \& Bluemner, E. (1980). Time series analysis: Clinical applications evaluating intervention effects in diaries. In O. D. Anderson (Ed.), Analysing time series: Proceedings of the international conference held on Guernsey, Channel Islands, October 1979 (pp. 291-312). Amsterdam: North-Holland.

Rissannen, J. (1978). Modeling the shortest data description. Automatica, 14, 465-471.

ROSEL, J., \& ElósEGUI, E. (1994). Daily an weekly smoking habits: A Box Jenkins analysis. Psychological Reports, 75, 1639-1648.

SAS InSTITUTE (1999). SAS/ETS (Version 8) [Software]. Cary, NC: Author.

SCHWARZ, G. (1978). Estimation the dimension of a model. Annals of Statistics, 6, 461-469.

SimonToN, D. K. (1977). Cross-sectional time-series experiments. Some suggested statistical analysis. Psychological Bulletin, 84, 489-502.

STADNYTSKa, T., BRAUN, S., \& WERNER, J. (2007). Model identification of integrated ARMA processes. Manuscript submitted for publication.

THORNTON, T. L., \& GILDEN, D. L. (2005). Provenance of correlation in psychological data. Psychonomic Bulletin \& Review, 12, 409-441.

Tsay, R. S., \& Tiao, G. C. (1984). Consistent estimates of autoregressive parameters and extended sample autocorrelation function for stationary and nonstationary ARMA models. Journal of American Statistical Association, 79, 84-96.

TSAY, R. S., \& TIAO, G. C. (1985). Use of canonical analysis in time series model identification. Biometrika, 72, 299-315.

TSAY, R. S., \& TIAo, G. C. (1990). Asymptotic properties of multivariate nonstationary processes with applications to autoregressions. Annals of Statistics, 18, 220-250.

VeLICER, W. F., \& COLBY, S. M. (1997). Time series analysis for prevention and treatment research. In K. J. Bryant, M. Windle, \& S. G. West (Eds.), The science of prevention: Methodological advances from alcohol and substance abuse research (pp. 211-249). Washington, DC: American Psychological Association.

VELICER, W. F., \& FAVA, J. L. (2003). Time series analysis. In J. Schinka \& W. F. Velicer (Eds.), Research methods in psychology (pp. 581-606). New York: Wiley.

VELICER, W. F., \& HARROP, J. W. (1983). The reliability and accuracy of time series model identification. Evaluation Review, 7, 551-560. 
VELICER, W.F., \& MCDONALD, R. P. (1984). Time series analysis without model identification. Multivariate Behavional Research, 19, 33-47.

VeuICER, W. F., \& MCDONALD, R. P. (1991). Cross-sectional time series design: A general transformation approach. Multivariate Behavional Research, 26, 247-254.

Velicer, W. F., Redding, C. A., Richmond, R., Greeley, J., \& SwIFT, W. (1992). A time series investigation of tree nicotine regulation models. Addictive Behaviors, 17, 325-345.

Velicer, W. F., Rossi, J. S., Diclemente, C. C., \& Prochaska, J. O. (1996). A criterion measurement model for health behavior change. Addictive Behaviors, 21, 555-584.

WAGENMAKERS, E.-J., FARRELL, S., \& RATCLIFF, R. (2004). Estimation an interpretation of $1 / f a$ noise in human cognition. Psychonomic Bulletin \& Review, 11, 579-615.

Williams, E. A., \& Gottman, J. M. (1999). A user's guide to the
Gottman-Williams time-series analysis computer programs for social scientists. Cambridge: Cambridge University Press.

\section{NOTE}

1. The spectral density function gives an amount of variance accounted for by each frequency we can measure. In time series analysis, the term frequency describes how rapidly things repeat themselves. The frequency domain analysis can be seen as a form of ANOVA where the overall variance of time series is divided into variance components due to independent cycles of different length.

(Manuscript received September 27, 2006; revision accepted for publication April 2, 2007.) 